\title{
Checklist de espécies de Polychaeta (Annelida) da Praia do Seixas, João Pessoa, Estado da Paraíba, Nordeste do Brasil
}

\section{Dimítri de Araújo Costa ${ }^{1}$, Hélder Formiga Fernandes', Francisco de Assis da Silva1, Martin Lindsey Christoffersen ${ }^{2}$}

${ }^{1}$ Programa de Pós-Graduação em Desenvolvimento e Meio Ambiente (PRODEMA). Universidade Federal da Paraíba. João Pessoa-PB. Brasil (CEP 58051-900). E-mail: costa.researcher@yahoo.com.br

${ }^{2}$ Departamento de Sistemática e Ecologia. Universidade Federal da Paraíba. João Pessoa-PB. Brasil (CEP 58051-900).

Resumo. O presente estudo apresenta a lista das espécies de poliquetas coletados na Praia do Seixas, município de João Pessoa, Estado da Paraíba, Nordeste do Brasil. Um total de 21 famílias, 36 gêneros e 49 espécies foram identificados. Os espécimes foram coletados durante o ano de 2015, bimestralmente, dentro de amostras de rodolitos. As Famílias Eunicidae e Cirratulidae foram as mais representativas. As espécies Cirriformia capensis, Dodecaceria capensis, Dorvillea angolana, Lysarete raquelae, Drilonereis falcata, Phyllodoce malmgreni, Synelmis sotoi, Lepidonotus spiculus, L. squamatus e Jasmineira caudata são consideradas novos registros para o Atlântico Sul. Além disso, 23 espécies são consideradas novos registros para a costa paraibana. O trabalho complementa o conhecimento da diversidade de poliquetas para a costa paraibana e suporte para futuros projetos de gestão ambiental.

Palavras-chave: João Pessoa; Novos registros; Rodolitos; Vermes marinhos.

Abstract. Checklist of species of Polychaeta (Annelida) of the Seixas Beach, João Pessoa, state of Paraíba, Northeast Brazil. This study presents the checklist of the species of polychaetes collected in Seixas Beach, Municipality of João Pessoa, State of Paraíba, Northeast Brazil. A total of 21 families, 36 genera, and 49 species were identified. The specimens were collected during the year of 2015, bimonthly, through samples of rhodoliths. The Families Eunicidae and Cirratulidae were most representative. The species Cirriformia capensis, Dodecaceria capensis, Dorvillea angolana, Lysarete raquelae, Drilonereis falcata, Phyllodoce malmgreni, Synelmis sotoi, Lepidonotus spiculus, L. squamatus and Jasmineira caudata are considered new records from South Atlantic. In addition, 23 species are considered new records from the Paraíba Coast. The work complements the knowledge of polychaetes diversity from Paraíba Coast and supports to future projects of environmental management.

Keywords: João Pessoa; New records; Rhodoliths; Marine worms.
Recebido:

04/09/2017

Aceito:

$18 / 11 / 2017$

Publicado:

$31 / 12 / 2017$

Acesso aberto Artigo completo

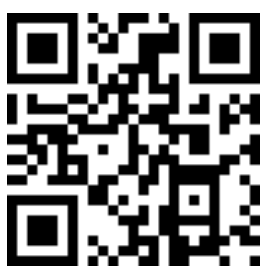

ORCIID

(ㄱ) 0000-0002-5399-2483 Dimítri de Araújo Costa

(D) 0000-0002-0370-0673

Hélder Formiga

Fernandes

(D) 0000-0003-4329-1672

Francisco de Assis da Silva

(D) $0000-0001-8108-1938$

Martin Lindsey

Christoffersen 


\section{Introdução}

O grupo Polychaeta Grube, 1850 abrange uma grande diversidade de organismos metamerizados, sendo característico de ambientes aquáticos, principalmente marinhos (Blake e Eugene, 2007). Os poliquetas apresentam uma ampla variação morfológica, podendo ser tubícolas ou errantes, permitindo-lhes adaptarem-se a diversos tipos de habitats, vivendo em substratos duros (consolidados e não consolidados) e moles, como recifes de corais, costões rochosos, estuários, lagoas costeiras, manguezais, praias, fundos arenosos, areno-lamosos e lamosos, bem como em águas continentais, ambientes terrestres e em aberturas hidrotermais no fundo do mar (Desbruyères e Toulmond, 1998; Nygren et al., 2013). Eles também são bastante comuns em rodolitos (algas calcárias vermelhas), podendo habitar dentro ou sobre esse tipo de substrato.

Atualmente, existem aproximadamente 9.000 espécies nominais de poliquetas (Rouse e Pleijel, 2001). Destas, de acordo com o último levantamento compilado por Amaral et al. (2013), há 1.149 espécies, 434 gêneros e 68 famílias reportados para a costa brasileira. Na Paraíba, existem 89 espécies registradas (De Assis et al., 2012).

$\mathrm{O}$ presente artigo contribui para aumentar o conhecimento taxonômico dos poliquetas para o Estado da Paraíba, como também para o Atlântico Sul, dando suporte para futuros projetos de gestão ambiental.

\section{Material e Métodos}

\section{Área de estudo}

Com cerca de $120 \mathrm{~km}$ de extensão, a costa da Paraíba apresenta clima tropical úmido, com uma baixa amplitude de temperatura, variando entre 24 a $27{ }^{\circ} \mathrm{C}$, umidade relativa do ar com médias anuais de $80 \%$, e precipitação entre 900 e 1.800 $\mathrm{mm}$ ao ano (Lima e Heckendorff, 1985; Costa, 2016). Esta região apresenta várias praias, as quais possuem várias características, como recifes de corais, ambientes arenosos, lamosos, além de ser recoberto por um banco de rodolitos, principalmente na Praia do Seixas, que é localizada no Município de João Pessoa. Esta praia é caracterizada por apresentar um crescente impacto antrópico, devido ao turismo nas regiões recifais, como também por meio da poluição influenciada pelos efluentes carreados pelo Rio do Cabelo.

As coletas foram realizadas bimestralmente nos ambientes recifais da Praia do Seixas, que varia entre 1,5 e 4,0 m de profundidade (Figura 1), durante o ano de 2015.

\section{Amostras}

As amostras são constituídas de rodolitos (Corallinophycidae, Rhodophyta), que são agregados de nódulos de algas vermelhas incrustantes (Figura 2).

O material coletado foi acondicionado em sacos plásticos com água do mar e etiquetas de identificação, sendo posteriormente levado ao Laboratório de Invertebrados Paulo Young (LIPY), do Departamento de Sistemática e Ecologia, da Universidade Federal da Paraíba (UFPB), para a realização da triagem dos poliquetas.

Em laboratório, as amostras foram mantidas separadamente em bandejas contendo água do mar, com salinidade 35 ppm e arejamento contínuo, até posterior processamento do material biológico, utilizando marreta e ponteiro. Os poliquetas foram triados sob lupa manual, com 0 auxílio de pinça e/ou pipeta Pasteur. Posteriormente, foram anestesiados em mentol, fixados em formalina a $4 \%$, diluído com água do mar, sendo finalmente preservados em álcool a 70\%. 


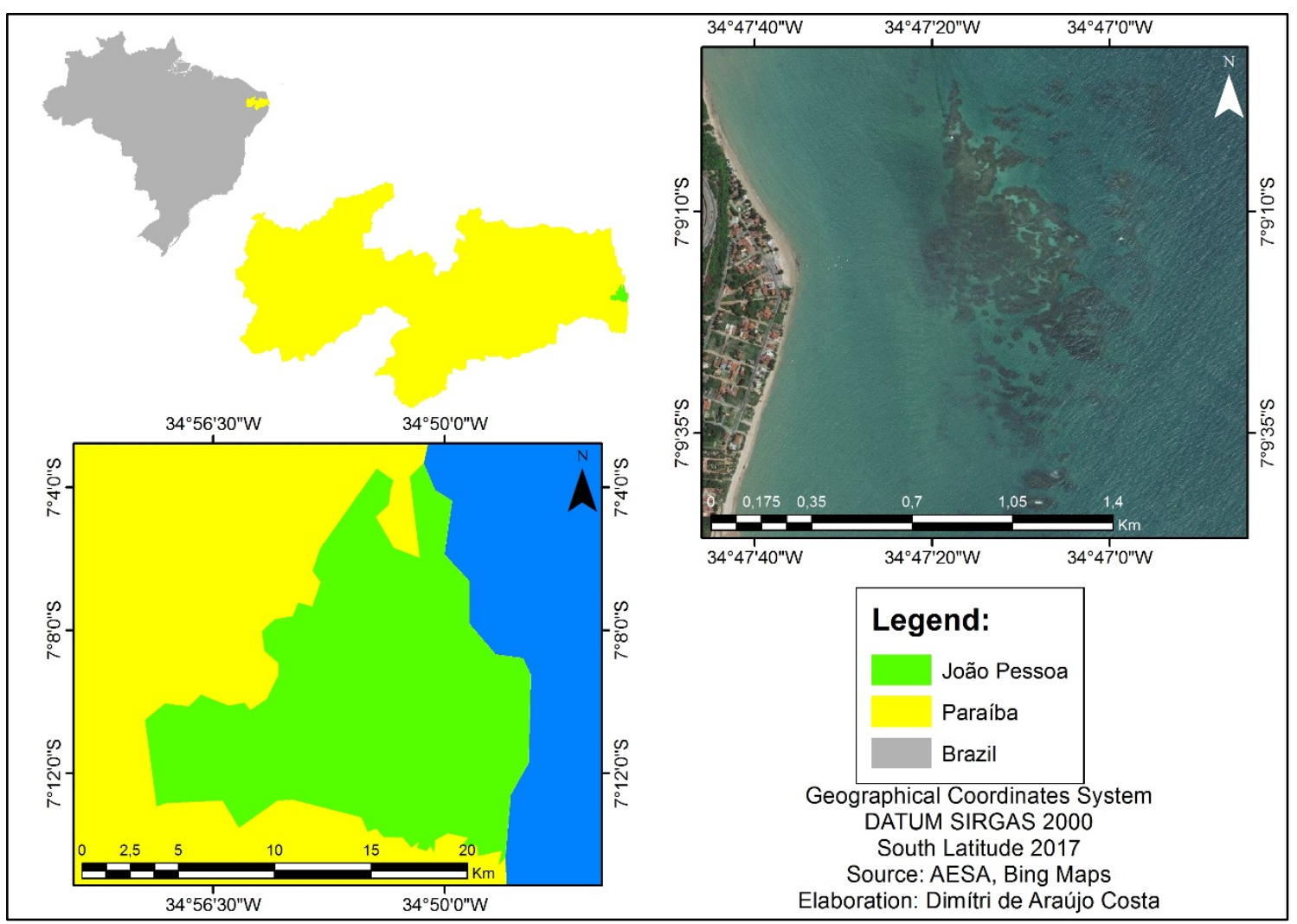

Figura 1. Mapa de localização dos ambientes recifais da Praia do Seixas, Paraíba.

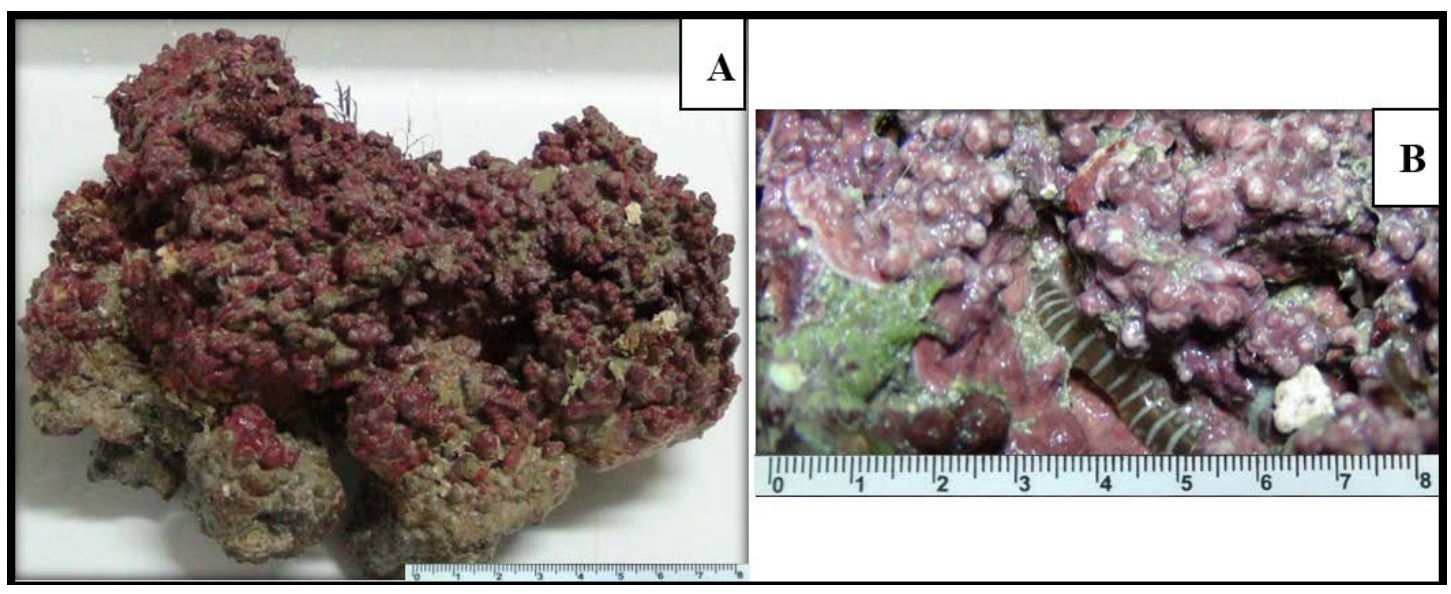

Figura 2. A- Visão geral de um agregado consolidado de rodolito. B- Poliqueta Hesione splendida rastejando entre os nódulos (Fotos: Dimítri A. Costa, 2015).

As identificações taxonômicas foram baseadas em Day (1967a, 1967b), Nonato e Luna (1970), Fauchald (1977, 1992), Uebelacker e Johnson (1984),
Amaral e Nonato (1994), Blake et al. (1995, 1996, 1997), Camargo e Lana (1995), Corrales (2003), Santos e Lana (2003), Viéitez et al. (2004), Barroso e Paiva 
(2007), De Assis et al. (2007), Costa et al. (2008) e Böggemann (2009). Todos os espécimes foram depositados na Coleção de Invertebrados Paulo Young (CIPY/DSEUFPB).

\section{Resultados}

Um total de 21 famílias, 36 gêneros e 49 espécies foram encontradas na Praia do Seixas, listadas na Tabela 1.

Tabela 1. Poliquetas coletados na Praia do Seixas, durante o ano de 2015, organizados de acordo com o sistema de classificação de Read (2016).

Errantia

Amphinomida

Amphinomidae

Eurythoe complanata

Eunicida

Dorvilleidae

Dorvillea angolana (Augener, 1918)

Eunicidae

Eunice atlantica Kinberg, 1865

Eunice biannulata Moore, 1904

Eunice filamentosa Grube \& Örsted in Grube, 1856

Eunice guanica (Treadwell, 1921)

Eunice imogena (Monro, 1924)

Eunice wasinensis Fauchald, 1992

Lysidice ninetta Audoin \& Milne Edwards, 1833

Lysidice unicornis (Grube, 1840)

Marphysa angelensis Fauchald, 1970

Marphysa regalis Verrill, 1900

Marphysa stylobranchiata Moore, 1909

Palola brasiliensis Zanol, Paiva \& Attolini, 2000

Lumbrineridae

Lumbrineris inflata Moore, 1911

Lumbrineris latreilli Audoin \& Milne Edwards, 1834

Lysarete raquelae Carrera-Parra, 2001

Oenonidae

Arabella iricolor (Montagu, 1804)

Drilonereis falcata Moore, 1911

Aphroditiformia

Polynoidae

Lepidonotus spiculus (Treadwell, 1906)

Lepidonotus squamatus (Linnaeus, 1758)

Nereidiformia

Chrysopetalidae

Bhawania obscura (Grube, 1868) 
Hesionidae

Hesione splendida Savigny in Lamarck, 1818

Oxydromus pugettensis (Johnson, 1901)

Syllidia amaralae Rizzo \& Salazar-Vallejo, 2014

Nereididae

Ceratonereis (Ceratonereis) singularis Treadwell, 1929

Nereis riisei Grube, 1857

Pseudonereis gallapagensis Kinberg, 1865

Pilargidae

Synelmis sotoi Salazar-Vallejo, 2003

Syllidae

Syllis guidae Nogueira \& Yunda-Guarin, 2008

Syllis prolifera Krohn, 1852

Phyllodociformia

Phyllodocidae

Phyllodoce malmgreni Gravier, 1900

Phyllodoce schmardaei Day, 1963

Sedentaria

Canalipalpata

Sabellida

Sabellariidae

Phragmatopoma caudata Krøyer in Mörch, 1863

Sabellidae

Branchiomma nigromaculatum (Baird, 1865)

Jasmineira caudata Langerhans, 1880

Terebellida

Cirratuliformia

Cirratulidae

Cirratulus africanus Gravier, 1906

Cirriformia capensis (Schmarda, 1861)

Dodecaceria capensis Day, 1961

Timarete punctata (Grube, 1859)

Flabelligeridae

Pherusa scutigera (Ehlers, 1887)

Terebellomorpha

Ampharetidae

Amphicteis gunneri (Sars, 1835)

Terebellidae

Terebella pterochaeta (McIntosh, 1885)

Terebella plagiostoma (Schmarda, 1861) 
Scolecida

Capitellidae

Capitella capitata (Fabricius, 1780)

Neopseudocapitella brasiliensis Rullier \& Amoureux, 1979

Maldanidae

Nicomache lanai De Assis, Samiguel \& Christoffersen, 2007

Orbiniidae

Naineris dendritica (Kinberg, 1867)

Naineris setosa (Verrill, 1900)

Das 49 espécies identificadas, 10 são novas ocorrências para o Atlântico Sul, Cirriformia capensis, Dodecaceria capensis, Dorvillea angolana, Lysarete raquelae, Drilonereis falcata, Phyllodoce malmgreni, Synelmis sotoi, Lepidonotus spiculus, L. squamatus e Jasmineira caudata. Além disso, 23 espécies são novas ocorrências para o estado da Paraíba, Amphicteis gunneri, Neopseudocapitella brasiliensis, Bhawania obscura, Cirraulus africanus, Eunice atlantica, E. biannulata, E. guanica, E. imogena, E. wasinensis, Marphysa angelensis, M. stylobranchiata, Pherusa scutigera, Syllidia amaralae, Lumbrineris inflata, Pseudonereis gallapagensis, Arabella iricolor, Naineris dendritica, N. setosa, P. schmardaei, Syllis guidae, S. prolifera, Terebella pterochaeta e T. plagiostoma.

\section{Discussão}

O Estado da Paraíba é um local que abriga vários tipos de invertebrados marinhos em sua costa litorânea, incluindo diversas espécies de poliquetas, os quais são organismos importantes para a indicação de poluição ambiental, sendo, portanto, denominados de bioindicadores (Amaral et al., 1998; Vieira et al., 2012).

Os rodolitos abrigam várias espécies de invertebrados, servindo de abrigo e refúgio (Scherner et al., 2010). Devido à grande quantidade de rodolitos na Praia do Seixas, foram encontrados diversos tipos de poliquetas, incluindo errantes e tubícolas. Dentre os errantes, destacam-se as espécies da Família Eunicidae, e dentre os tubícolas as mais representativas são as espécies registradas da Família Terebellidae.

\section{Conclusão}

O presente trabalho contribui para o conhecimento da fauna de poliquetas do Estado da Paraíba, que juntamente com o trabalho de De Assis et al. (2012), são agora registradas 122 espécies. Desta forma, das espécies de poliquetas registradas para o Brasil cerca de $10 \%$ são reportadas para a costa paraibana.

\section{Agradecimentos}

Os autores agradecem ao apoio institucional e acadêmico fornecido pela Universidade Federal da Paraíba, através do Laboratório de Invertebrados Paulo Young, do Departamento de Sistemática e Ecologia, como também pela Coordenação do Programa de Pós-Graduação em Desenvolvimento e Meio Ambiente (PRODEMA), por meio do Prof. Dr. Reinaldo Farias Paiva de Lucena. D. A. C. agradece à bolsa de doutorado recebida pela Fundação de Apoio à Pesquisa do Estado da Paraíba (FAPESq) em parceria com a Coordenação de Aperfeiçoamento do Ensino Superior (CAPES). M. L. C. agradece pela bolsa de produtividade fornecida pelo Conselho Nacional de Desenvolvimento Científico e Tecnológico (CNPq). 


\section{Declaração de conflito de interesses}

Os autores declaram não haver conflito de interesses.

\section{Referências}

Amaral, A. C. Z.; Morgado, E. H.; Salvador, L. B. Poliquetas bioindicadores de poluição orgânica em praias paulistas. Revista Brasileira de Biologia, v. 58, p. 307-316, 1998.

Amaral, A. C. Z.; Nallin, S. A. H.; Steiner, T. M.; Forroni, T. O.; Gomes Filho, G. Catálogo das espécies de Annelida Polychaeta do Brasil. 2013. Disponível em: <http://www.ib.unicamp.br/museu_zoologia/site s/www.ib.unicamp.br.museu_zoologia/files/Cat \%C3\%A1logo_Polychaeta_Brasil_Amaral_et_a 1_2013_1a.pdf>. Acesso em: 25 mar. 2015.

Amaral, A. C. Z.; Nonato, E. F. Anelídeos poliquetos da costa brasileira. 5. Pisionidae, Chrysopetalidae, Amphinomidae e Euphrosinidae. Revista Brasileira de Zoologia, v. 11, n. 2, p. 361-390, 1994.

Barroso, R.; Paiva, P. C. Amphinomidae (Annelida: Polychaeta) from Rocas Atoll, Northeastern Brazil. Arquivos do Museu Nacional, v. 65, n. 3, p. 357-362, 2007.

Blake, J. A.; Eugene, R. R. Polychaeta. In: Carlton, J. T. (Ed). The Light and Smith Manual: Intertidal invertebrates from Central California to Oregon. Berkeley: University of California Press, 2007. p. 309-410.

Blake, J. A.; Hilbig, B.; Scott, P. H. Taxonomic atlas of the benthic fauna of the Santa Maria basin and the Western Santa Barbara Channel. Volume 4. The Annelida Part 1, Oligochaeta and Polychaeta: Phyllodocida (Phyllodocidae to Paralacydoniidae). rev. ed. Santa Barbara: Santa Barbara Museum of Natural History, 1997.

Blake, J. A.; Hilbig, B.; Scott, P. H. Taxonomic atlas of the benthic fauna of the Santa Maria Basin and the Western Santa Barbara Channel. Volume 5. The Annelida Part 2, Polychaeta: Phyllodocida (Syllidae and scalebearing families), Amphinomida, and Eunicida. Santa Barbara: Santa Barbara Museum of Natural History, 1995.

Blake, J. A.; Hilbig, B.; Scott, P. H. Taxonomic atlas of the benthic fauna of the Santa Maria Basin and the Western Santa Barbara Channel. Volume 6. The Annelida Part 3, Polychaeta: Orbiniidae to Cossuridae. Santa
Barbara: Santa Barbara Museum of Natural History, 1996.

Böggemann M. Polychaetes (Annelida) of the abyssal SE Atlantic. Organisms Diversity \& Evolution, $\quad$ v. 9, $\quad$ p. 251-428, 2009. https://doi.org/10.1016/j.ode.2009.10.001

Camargo, M. G; Lana, P. C. Lumbrineridae (Polychaeta: Eunicemorpha) da costa sul e sudeste do Brasil. II. Lumbrineris. Iheringia, Série Zoologia, v. 79, p. 93-120, 1995.

Corrales, M. C. Estudio de la criptofauna coralina y dos anélidos poliquetos (Annelida: Polychaeta) de substratos duros del Parque Nacional de Coiba, Panamá. Madrid: Universidad Autónoma de Madrid, 2003. (Tese de Doutorado).

Costa, D. A. Assembleias de poliquetas (Annelida), associadas aos rodolitos (Corallinophycidae, Rhodophyta), na Praia do Seixas, João Pessoa, Paraíba, Brasil. Rio Tinto: Universidade Federal da Paraíba, 2016. (Dissertação de mestrado).

Costa, D. A.; De Assis, J. E.; Christoffersen, M. L. New synonym of Hesione splendida (Hesionidae, Polychaeta, Annelida). Biociências, v. 16, n. 2, p. 131-133, 2008. Disponível em: <http://revistaseletronicas. pucrs.br/fabio/ojs/index.php/fabio/article/view/6 503/4716>. Acesso em: 25 mar. 2015.

Day, J. H. A monograph on the Polychaeta of Southern Africa, Part 1. Errantia. London: Trustees of the British Museum of Natural History, 1967a.

Day, J. H. A monograph on the Polychaeta of Southern Africa, Part 2. Sedentaria. London: Trustees of the British Museum of Natural History, 1967b.

De Assis, J. E.; Alonso C.; Brito, R. J.; Santos, A. S.; Christoffersen, M. L. Polychaetous annelids from the coast of Paraíba State, Brazil. Revista Nordestina de Biologia, v. 21, n. 1, p. 3-45, 2012. Disponível em: <http://periodicos.ufpb.br/index.php/revnebio/ar ticle/view/13625/8527>. Acesso em: 25 mar. 2015.

De Assis, J. E.; Alonso, C.; Christoffersen, M. L. Two new species of Nicomache (Polychaeta: Maldanidae) from the Southwest Atlantic. Zootaxa, v. 1454, p. 27-37, 2007. http://dx.doi.org/10.11646/zootaxa.1454.1.2

Desbruyères, D.; Toulmond, A. A new species of hesionid worm, Hesiocaeca methanicola sp. nov. (Polychaeta: Hesionidae), living in ice-like methane hydrates in the deep Gulf of Mexico. 
Cahiers de Biologie Marine, v. 39, n. 1, p. 9398, 1998.

Fauchald, K. Polychaetes from intertidal areas in Panama, with a review of previous shallowwater records. Smithsonian Contributions to Zoology, v. 221, $\quad$ p. 1-81, 1977. https://doi.org/10.5479/si.00810282.221

Fauchald, K. A review of the genus Eunice (Polychaeta: Eunicidae) based upon type material. Smithsonian Contributions to Zoology, v. 523, p. 1-422, 1992. https://doi.org/10.5479/si.00810282.523

Lima, P. J.; Heckendorff, W. D. Climatologia. In: Governo do Estado da Paraíba, Secretaria da Educação, Universidade Federal da Paraíba. (Eds). Atlas Geográfico do Estado da Paraíba. João Pessoa: Grafset, 1985. p. 34-44.

Nonato, E. F.; Luna, J. A. C. Anelídeos poliquetas do Nordeste do Brasil. I - Poliquetas bentônicos da costa de Alagoas e Sergipe. Boletim do Instituto Oceanográfico, v. 19, p. 57-130， 1970. http://dx.doi.org/10.1590/ S0373-55241970000100004

Nygren, A.; Hall, R.; Pleijel F. Bestämningsnyckel till och presentation av svenska familjer av havsborstmaskar. 2013. Disponível em: <http://www.slu.se/ Global/externwebben/centrumbildningarprojekt/artdatabanken/Dokument/Publikationer/ Polychaeta.pdf>. Acesso em: 25 mar. 2015.

Read, G. Polychaeta. World Register of Marine Species. 2016. Disponível em: $<$ http://www.marinespecies.org/aphia.php?p=ta xdetails\&id=883> . Acesso em: 15 out 2017.
Rouse, G. W.; Pleijel F. Polychaetes. Oxford: Oxford University Press, 2001.

Santos, C. S. G.; Lana, P. C. Nereididae (Polychaeta) da costa Nordeste do Brasil. III. Gêneros Ceratonereis e Nereis. Iheringia, Série Zoologia, v. 93, $\quad$ n. 1, p. 5-22, 2003. https://doi.org/10.1590/S007347212003000100001

Scherner, F.; Riul, P.; Bastos, E.; Bouzon, Z. L.; Pagliosa, P. R.; Blankensteyn, A.; Oliveira, E. C.; Horta, P. A. Herbivory in a rhodolith bed: a structuring factor? Pan-American Journal of Aquatic Sciences, v. 5, p. 358-366, 2010. Disponível em: <http://www.panamjas.org/ pdf_artigos/PANAMJAS_5(3)_358-366.pdf>. Acesso em: 23 set. 2016.

Uebelacker, J. M.; Johnson, P. G. Taxonomic guide to the polychaetes of the Northern Gulf of Mexico. Mobile: Barry A. Vittor \& Associates, 1984. (Final report to the Minerals Management Service).

Vieira, J. V.; Borzone, C. A.; Lorenzi, L.; De Carvalho, F. G. Human impact on the benthic macrofauna of two beach environments with different morphodynamic characteristics in Southern Brazil. Brazilian Journal of Oceanography, v. 60, n. 2, p. 135-148, 2012. https://doi.org/10.1590/S167987592012000200004

Viéitez, J. M.; Alós, C.; Parapar, J.; Besteiro, C.; Moreira, J.; Núñez, J.; Laborda, A. J.; San Martín, G. Fauna Ibérica: Annelida Polychaeta I. Madrid: Museo Nacional de Ciencias Naturales, 2004. v. 25.

Informação da Licença: Este é um artigo Open Access distribuído sob os termos da Licença Creative Commons Attribution, que permite uso irrestrito, distribuição e reprodução em qualquer meio, desde que a obra original seja devidamente citada. 